\title{
Comparison Single Embryo Transfer (SET) and Fetal Reduction (Three to One) Outcomes of Pregnant Women After Fetal Reduction
}

Yue-Lin Fang ${ }^{1}$, I-Fang Yang ${ }^{1}$, Wei-Jiun Li ${ }^{1}$, Lee-Wen Huang ${ }^{1}$, JierZen Chang Hun-Shan'1, Pan Kenneth Pan², Cristiana Pan², Li-Che $\mathrm{Lu}^{1}$ and Yi-Ho Cheng ${ }^{1 *}$

\section{Institutional affiliations}

${ }^{1}$ Obstetrics and Gynecology Department, Shin Kong Wu Ho-Su Memorial Hospital, Taipei, ROC

${ }^{2}$ Science Department, University of British Columbia, Canada

\section{Correspondence Address}

General surgery Department,

Shin Kong Wu Ho-Su Memorial Hospital, 111 Shilin,

Taipei, Taiwan, ROC.

Tel: +886-2-28332211.

E-mail:pan5511@me.com

Received Date: Nov 10, 2020

Accepted Date: Nov 11, 2020

Published Date: Dec 11, 2020

Keywords: Birth outcomes; Cerebral palsy; Corticosteroid therapy; Multifetal pregnancy reduction; Pregnancy

\section{Background and Purpose}

Compared with multiple births, single embryo transfer pregnancies are not as high as risk of significant child morbidity, particularly cerebral palsy, after high-order fetal reduction. This is termed the vanishingtwin syndrome, and is an important etiology of cerebral palsy. Antenatal corticosteroid therapy (CCT) is widely used during preterm labor to enhance lung maturity, the use of dexamethasone, however, increase the risk for detrimental long-term neurodevelopmental effects. Thus, in this study we assess the effect of CCT on neurological disability and congenital abnormality in SET and control group after fetal reduction and over 2 years of age.

\section{Methods}

The CCT (38 sets of SET) and control (56 sets) groups were compared for the incidences of cerebral palsy and congenital abnormalities, as well as mean birth weight, maternal age, and mean gestational age at delivery and at fetal reduction using records from Shin-Kong Memorial Hospital for the period 2008-2017.

\section{Results}

There were 94 quadruplet-reduced sets of SET in our study. All of these SET were alive and aged over 2 years at the time of this writing. The gestational age at the time of delivery was $39+-2.4$ weeks for the SET group vs. $36 \pm 2.9$ weeks for the controls, with mean birth weights of $2798 \pm 555 \mathrm{~g}$ vs. $2685 \pm 519 \mathrm{~g}$, respectively $(\mathrm{p}<0.01)$. All 94 sets of twins were delivered by cesarean section, with only 1 case of cerebral palsy in Control group, while the others had developed normally by the age of 2 years. Only mean birth weight and gestational age at delivery showed significant differences in comparisons of the 2 groups of twins.

\section{Conclusion}

The reasons why IVF treatment sometimes results in twin or triplet births - even though only one embryo is used - have been found by the largest study to date on single embryo transfer (SET) and multiple pregnancy. However, despite performing SET, multiple pregnancies do happen due to a phenomenon known as 'zygotic splitting', when one embryo divides resulting in twins or triplets. It is more prevalent following SET than in spontaneous conception.

The reasons for this were unclear until now. The new study identified risk factors for zygotic splitting following SET. The most significant factors were: using blastocyst culture whereby embryos are matured in the lab for several days prior to implantation; assisted hatching whereby a small hole is made in a layer of proteins surrounding the embryo to help its attachment to the uterine wall; and the use of frozen-thawed embryos rather than fresh embryos. The study, published in Human Reproduction, analyzed data from 937,848 cycles of SET from the Japanese assisted reproductive technology national registry database. The researchers were able to identify the true cases of zygotic splitting by counting the births in which the number of fetuses outweighed the number of gestational sacs (a large fluid cavity surrounding an embryo). 'Big data makes a big difference,' said Professor Peter Braude, emeritus professor of obstetrics and gynaecology at King's College, London, who was not involved in the research. The true prevalence of zygotic splitting was calculated to be just 1.36 percent, which is 'reassuring' according to Professor Braude. Although some embryo manipulation techniques were identified as risk factors for zygotic splitting, Dr Kuroda also pointed out that technologies have been improving. 'The use of single embryo transfer has increased worldwide, the prevalence of zygotic splitting has not,' he said. 'This may be because ART techniques and also the cultures in which blastocysts are matured in the lab, have improved over recent years, reducing the stress on the embryo and leading to a decrease in the risk of zygotic splitting.' He added: 'So, there may be no need to avoid embryo manipulations such as blastocyst culture, in order to select the single most viable embryo. 'The present report summarizes follow-up findings for a sample population of 2-year-old fetal-reduction twins enrolled in a prospective study of antenatal CCT to evaluate birth outcomes. Glucocorticoids have been used for many years to enhance lung maturity in neonates. One study [5] described the use of corticosteroids to reduce both the incidence and severity of neonatal respiratory distress syndrome. Since then, the beneficial and adverse effects of CCT have been widely discussed [2,6-9]. Multiple CCT remains a controversial issue with respect to birth outcomes. Recently, Evans MI et al. [10] reported that antenatal dexamethasone treatment is associated with periventricular leukomalakia due to the neurotoxic effects of the corticosteriod's sulphite preservatives. Futher, Shinwell et al. [2] stated that a 3-day course of CCT in preterm infants was associated with a significantly increased incidence of cerebral palsy and developmental delay. said study author Dr Keiji Kuroda of the Sugiyama Clinic Shinjuku and Juntendo University Faculty of Medicine in Tokyo, Japan.

Transferring just one embryo at a time is recommended to reduce rates of multiple pregnancies from IVF, as such pregnancies pose risks to 


\section{Journal of Clinical Obstetrics and Gynecology Research}

both mothers and babies; Therefore, Murphy et al. [11] concluded that the lack of firm evidence that antenatal corticosteroids are beneficial and the concern about potential adverse effects on fetal growth mean that the widespread administration of repeated courses of steroids to women with twin pregnancies may do more harm than good. The incidence of multiple pregnancies has increased dramatically since the introduction of an improvements in assisted reproductive techniques. Despite advances in perinatal and neonatal medicine, however, the mortality and morbidity associated with multifetal pregnancies is between 3 and 10 times that of the single variant [12]. Therefore, MPR was specifically developed to reduce complications associated with higher-order multiple gestations by actually decreasing fetal number [13]. Leviton and Gilles [14] suggested that remnant fetal tissues may theoretically gain access to the circulation of the remaining fetues, leading to fluctuations in fetal blood pressure and cerebral flow and, thus, initiating periventricular leukomalacia. The Japan Society of Obstetrics and Gynaecology recommended the use of SET in 2008, and by 2015 usage had increased by 80 percent and the prevalence of multiple pregnancies from IVF dropped from 10.7 to 3.2 percent. Eli Geva et al. [15] provided further confirmation of the theory that MPR might be an additional risk factor for periventicular leukomalacia among premature infants, regardless of twinning. Additionally, premature infants who have periventicular leukomalacia may be prone to the development of spastic diapiresis, the most common type of cerebral palsy [16]. This mechanism could be the result of an intrauterine inflammatory process caused by fetal death, which may initiate the production and release of inflammatory cytokines and induce thromboembolization [3]. Our preliminary results showed a trend towards a higher incidence of poor birth outcomes in the dexamethasone-treated group. Studies using larger sample sizes may be able to more-accurately determine the relationship between dexamethasone treatment after fetal reducation and birth outcomes. In our study, we determined only 2 significant differences in mean gestational age at delivery and mean birth weight for the CCT group. The impact on low birth weight may have occurred due to the interval from steroids exposure to the time of delivery or preterm birth. Therefore, strategies for prevention of low birth weight may be important for further reduction of perinatal mortality and the handicaps related to low birth weight. As a result of our findings, clinicians may want to consider whether they should counsel couples about the small increase in the risk of multiple pregnancies, as a result of zygotic splitting associated with some embryo manipulations,' increasing rates of maternal mortality, preterm births and low birth weight. Thus, limiting the number of embryo transfers is essential for preventing the adverse effects on birth outcomes. Despite the limitations of this investigation, it seems reasonable to suggest that our results indicate that caution with respect to limiting fetal numbers and CCT is entirely appropriate. We suggest that strict control of embryo quality and limitation of the number of embryo transfers be mandatory. Where reduction has been performed, a prolonged gestational age at delivery is important for the twin babies or single delivery. pregnancy after laparoscopic oocyte recovery, in-vitro fertilization and embryotransfer (author's transl). Geburtshilfe Frauenheilkd. 1982;42(3):197.

4. Kerin J, Quinn P, Kirby C, Seamark R, Warnes G, Jeffrey R, et al. Incidence of multiple pregnancy after in-vitro fertilisation and embryo transfer. Lancet. 1983;322(8349):537-40.

5. Tummers $P$, De Sutter P and Dhont M. Risk of spontaneous abortion in singleton and twin pregnancies after IVF/ICSI. Hum Reprod. 2003;18(8):1720-3.

6. Callahan TL, Hall JE, Ettner SL, Christiansen CL, Greene MF, Crowley WF Jr. The economic impact of multiple-gestation pregnancies and the contribution of assisted-reproduction techniques to their incidence. N Engl J Med. 1994;331(4):244-9.

7. Alpha Scientists in Reproductive Medicine. The Alpha consensus meeting on cryopreservation key performance indicators and benchmarks: proceedings of an expert meeting. Reproductive BioMedicine Online. 2012;25(2):146-67.

8. Ambroggio J, Gindoff PR, Molina B, Dayal M, Khaldi R, Peak D, et al. Multinucleation of a sibling blastomere on day 2 suggests unsuitability for embryo transfer in IVF - pre-implantation genetic screening cycles. Fertility \& Sterility. 2011;96:856-9.

9. Antezak M and Van Blerkom J. Temporal and spatialects of fragmentation in early human embryos: possible effects on developmental competence and association with the differential elimination of regulatory proteins from polarized domains. Human Reproduction. 1999;14:429-47.

10. Aparicio B, Cruz M, and Mesegeur M. Is Morphokinetic Analysis the Answer? Reproductive BioMedicine Online. 2013;27:654-63.

11. Templeton A and Morris JK. Reducing the risk of multiple births by transfer of two embryos after in vitro fertilization. N Engl J Med. 1998;339:573-577.

12. Coetsier $\mathrm{T}$ and Dhont $\mathrm{M}$. Avoiding multiple pregnancies in in-vitro fertilization: who's afraid of single embryo transfer? Hum Reprod. 1998;13(10):2663-4.

13. Strandell A, Bergh C and Lundin K. Selection of patients suitable for one-embryo transfer reduces the rate of multiple births by half without impairment of overall birth rates. Hum Reprod. 2000;15(12):2520-5.

14. Gerris J, De Neuborg D, Mangelschots K, Van Royen E, Van de Meerssche $M$ and Valkenburg M. Prevention of twin pregnancy after in-vitro fertilization or intracytoplasmic sperm injection based on strict embryo criteria: a prospective randomized clinical trial. Hum Reprod. 1999;14(10):2581-7.

15. Martikainen H, Tiitinen A, Tomas C, Juha T, Mauri O, Leena T, et al. One versus two embryo transfer after IVF and ICSI: a randomized study. Hum Reprod. 2001;16(9):1900-3.

16. Steer CV, Mills CL, Tan SL, Campbell S and Edwards RG. The cumulative embryo score: a predictive embryo scoring technique to select the optimal number of embryos to transfer in an invitro fertilization and embryo transfer programme. Hum Reprod. 1992;7(1):117-9.

1. Muasher S, Wilkes C, Garcia J and Rosenwaks Z, Jones H. Benefits and risks of multiple transfer with in vitro fertilisation. Lancet. 1984;323(8376):570.

2. Craft I, Porter R, Green S, Tucker M, Smith B, Twigg H, et al. Success of fertility, embryo number, and in-vitro fertilisation. Lancet. 1984;323(8379):732.

3. Feichtinger W, Szalay S, Kemeter P, Beck A and Janisch H. Twin 